\title{
La comunicación organizacional vista desde México
}

\author{
A comunicação organizacional desde a perspectiva do México \\ Organizational communication a vision from Mexico
}

\section{(9) Cristina Barroso Camiade}

- Dra. en Comunicación Aplicada por La Universidad Anáhuac México-Norte.

- Profesora de la Universidad Anáhuac México-Norte.

- E-mail: barrosocriss@gmail.com

Eva María Pérez Castrejón

Dra. en Comunicación Aplicada por La Universidad Anáhuac México-Norte.

Profesora de la Universidad Popular Autónoma del Estado de Puebla (Upaep).

- E-mail: evamaria.perez@upaep.mx 


\section{Resumen}

En este artículo se presenta el desarrollo de la disciplina en el tiempo para conocer los aportes teóricos más importantes que se han venido desarrollando en México, y asípor medio del caso de la Universidad Popular Autónoma del Estado de Puebla (Upaep) exponer las principales áreas de oportunidad que tiene la disciplina dentro de la vida académica y profesional de los egresados como una forma de entrelazar el desarrollo teórico con la práctica universitaria.

\section{PALABRAS CLAVE: COMUNICACIÓN ORGANIZACIONAL・MÉXICO・CONCEPTUALIZACIÓN・ENSEÑANZA•UPAEP.}

\section{Resumo}

Neste artigo é apresentado o desenvolvimento da disciplina ao longo do tempo para conhecer as contribuições teóricas mais importantes que foram desenvolvidas no México e, com isso, por meio do caso da Universidade Autônoma Popular do Estado de Puebla (Upaep), expor as principais áreas de oportunidade que têm a disciplina dentro da vida acadêmica e profissional dos egressos, com o objetivo de entrelaçar o desenvolvimento teórico com a prática universitária.

\section{PALAVRAS-CHAVE: COMUNICAÇÃO ORGANIZACIONAL・MÉXICO・CONCEITUALIZAÇÃO・ENSINO・UPAEP.}

\section{Abstract}

This article presents the development of organizational communication over time in order to recognize the most important theoretical contributions that have been developed in Mexico and, thus, from the case of the Popular Autonomous University of Puebla (Upaep), the article also seeks to expose the main areas of opportunity that the discipline has within the academic and professional life of the graduates as a way to intertwine the theoretical development with the university praxis. 


\section{LA COMUNICACIÓN ORGANIZACIONAL EN MÉXICO: DESARROLLO DE LA DISCIPLINA}

a comunicación organizacional (CO) en México se ha ido consolidando en la historia como una disciplina cuyas tendencias han variado, pero siempre han sido sustentadas desde la investigación. Dentro de la CO se ponen en práctica las competencias verbales y no verbales, la ética, el promover que las organizaciones sean socialmente responsables, la búsqueda de finanzas sanas, el mantenimiento de una buena relación con los medios de comunicación y lo más importante es que el especialista de esta disciplina siempre busca que los objetivos organizacionales que nacen y se desarrollan desde la cultura organizacional puedan ser alcanzados (Pérez Castrejón, 2017). De acuerdo a lo que establecen Tanius Karam y Andrés Cañizáles (2010), la comunicación organizacional necesariamente debe percibirse de manera integral y no de manera reduccionista como medios de comunicación internos.

A pesar de que la comunicación organizacional en México sigue en proceso de desarrollo, cada día aumenta el número de instituciones interesadas en verla como una posibilidad de crecimiento; para los especialistas de la comunicación, este tipo de comunicación no solo se practica y se posibilita dentro de la organización, sino también fuera de ésta. El buen funcionamiento de las redes internas debe ser paralelo a las que se tiene con el cliente externo (Guzmán Paz, 2012). Por lo que es importante ver si la CO se ha convertido en disciplina o indisciplina, para esto se hace un recorrido que contextualiza la problemática científica que han atravesado los estudios de la comunicación proyectada como una disciplina; una información cronológica desde las ciencias sociales, la comunicación en México y América Latina (De la Peza Casares, 2012).

Asimismo, la Comunicación Organizacional en México ha demostrado que es posible que las organizaciones tanto públicas como privadas sean capaces de cumplir con sus metas, si favorecen el desarrollo de la comunicación estratégica en su interior. Lo anterior, lo hace posible el especialista que se forma desde el aula y que posteriormente en la práctica es quien aprende y desarrolla las habilidades, que lo facultan para diagnosticar y generar propuestas que mantienen las buenas prácticas, pero que también rompen con paradigmas, esto, por medio de planes estratégicos de comunicación que después lo llevan a medir los resultados de los objetivos planteados. Previo a las propuestas que elabora el especialista, se realiza una lectura en materia de comunicación organizacional, interpreta y traduce las necesidades, ante grupos multiculturales y la necesidad de inclusión, buscando respuestas inmediatas por intermedio de las nuevas tecnologías y los diálogos con y entre los grupos que integran la organización y que son parte fundamental de la reputación organizacional en el ámbito interno y externo (Pérez Castrejón, 2017, p.278-296).

El estudio de la CO en México se ha enfocado en la diversidad de organizaciones, desde las grandes empresas hasta las pequeñas, lo que permite saber que el estudio de fenómenos organizacionales no siempre se aborda desde una misma perspectiva lo que ha llevado al país a una innovación organizacional donde existe la posibilidad de crear nuevas ideas que posteriormente se trasladan a productos, servicios, procesos y formas organizacionales. La innovación en las organizaciones es un tema de gran interés en el espacio académico, en que su percepción conceptual se aborda de manera diferente (Rodríguez Olaya; De la Rosa Alburquerque; Contreras Manrique, 2013).

La Comunicación Organizacional en México debe tener en cuenta la disposición de un sistema de gestión del conocimiento que sea puesto en práctica con la intención de que los miembros puedan crear, transferir e integrar conocimientos al servicio común donde se asegure el diálogo abierto y argumentativo entre los miembros y las partes. Esto requiere de un lenguaje común que permita un espacio de encuentro transparente, abierto y comprometido a la hora de ejercer sus funciones en la evolución de la organización como parte de un todo (Queris Rojas; Almirall Cabrera; Capote García; Alfonso Robayna, 2012).

El lenguaje común en la disciplina de la Comunicación Organizacional es creado o impulsado por los especialistas del área en México, quienes no pueden prescindir de conocimientos como comunicación estratégica, filosofía y cultura organizacional, 
ANO 16 • NÚMERO 30 • 1은. 2019 • ORGANICOM

LA COMUNICACIÓN ORGANIZACIONAL VISTA DESDE MÉXICO

jerarquía, manejo de crisis, fomentar las mejores relaciones cara a cara y entre subsistemas, trabajo en equipo y dinámicas de grupos, interculturalidad, ambiente, clima, identidad e imagen, recompensa y motivación, comunicación humana, temas referentes a capacitación, talleres, finanzas, comunicación productiva, ética y Responsabilidad Social Organizacional (Pérez Castrejón, 2017, p.278-296).

\section{PRINCIPALES PLANTEAMIENTOS TEÓRICOS DESARROLLADOS EN MÉXICO}

La importancia de la comunicación en las organizaciones radica en los gremios de seres humanos que se vinculan de manera congruente y ordenada con funciones específicas para la sociedad. La comunicación en las organizaciones públicas y privadas en México ha ido a la par de una evolución teórica en el contexto de las organizaciones que han pasado por diferentes etapas e intervenciones propias del fenómeno de la comunicación (Arellano Reinoso, 2014), en que el especialista aprende conocimientos que van desde la escritura, la historia, la psicología, finanzas y estadística, la organización y el manejo de crisis, lenguajes verbales y no verbales, de aspectos geopolíticos, entre muchos otros. Además, el especialista de la Comunicación Organizacional adquiere conocimientos teóricos para trabajar de manera interdisciplinaria con áreas tales como mercadotecnia, publicidad, desarrollo organizacional y recursos humanos entre otras.

Las aportaciones teóricas se ofrecen desde diferentes perspectivas, por ejemplo para Rebeil Corella (2006), la CO puede verse desde una perspectiva de comunicación integral que para las organizaciones es un proceso de índole social que reside en la producción, procesamiento e intercambio de información, que internamente y desde un sistema económico, político, cultural o social se da a la tarea de hacer fluir y acelerar mensajes e interacciones entre todos sus grupos de interés, desde tres puntos: público interno y externo, comunicación corporativa y relaciones públicas y la comunicación mercadológica, que anteriormente se estudiaban por separado. Hoy con el firme interés de ubicarse efectivamente en el mercado, las organizaciones establecen su importancia e impacto ante diferentes contextos que necesitan una comunicación sinérgica.

Igualmente, esta misma autora menciona que la Comunicación Organizacional puede coadyuvar a la mejora de las organizaciones sin ser una solución exclusiva de las problemáticas, ya que permite diseñar un traje a la medida que puede ser aplicado en organizaciones grandes o pequeñas y de cualquier giro (Rebeil Corella, 2006), es decir, es parte de lo que el especialista puede hacer, sumado a que dicho experto es el encargado de potenciar la contribución de cada una de las personas que conforman las organizaciones, en sus aportaciones tanto en la operación como en el pensamiento, para hacer a la organización más productiva a partir de la solución de problemas (Rebeil Corella; Ruizsandoval Reséndiz, 2000).

La CO sistematiza las relaciones de la organización dentro y fuera de su entorno; esto, como una contestación a la creciente demanda de los públicos que solicitan organizaciones que tengan credibilidad y atenúen confianza, lo que permite que la organización tenga una imagen organizacional positiva (Tejeda Palacios, 1987).

En México, generalmente quien estudia la disciplina de la Comunicación Organizacional tiene como referente a Nosnik Ostrowiak (2015), quien menciona que la comunicación en las organizaciones es una noción más profunda que debe cultivarse a partir de la teoría de los sistemas, es decir, que las organizaciones les deben ser vistas como una representación sistémica. Nosnik Ostrowiak explica que la CO requiere apertura, evolucionar y no ser rígida, ser multidimensional e instrumentada, es decir, la comunicación dentro de una organización siempre debe ser hacia el exterior para que los mensajes le lleguen al público, que sea oportuna, formal e informal, que sea de arriba hacia abajo y viceversa y que utilice diferentes herramientas para lograrlo.

El realizar acciones propias de la Comunicación Organizacional es una necesidad para todas las organizaciones y va más allá de las finanzas y asuntos técnicos, esta disciplina tiene que ver con la satisfacción de las personas que integran la 
organización y la hacen viva para hacer de ésta un sistema competente ante las demandas sociales, por lo que en otro punto de vista, la CO se ve a partir de los valores y principios con los que se rige la cultura de cada sistema, lo que posibilita permanentemente la mejora de las organizaciones, no con la intención de juzgar, sino para favorecer a quienes integran la organización y con quienes se vincula e interrelaciona ésta por medio de sus valores, recursos, misión, cultura, su visión y sus relaciones con el exterior. En este análisis, las organizaciones públicas y privadas se concentran en la adecuada tarea del manejo del conocimiento, de la información y de las interrelaciones (Peiró Silla; Bresó Peiró, 2012).

Tomando en cuenta las aportaciones de los diferentes autores, se puede comprender a la comunicación organizacional, hoy llamada comunicación corporativa, como la base del buen trabajo de las organizaciones, que contribuye a que los mensajes lleguen de manera correcta y puedan ser retroalimentados. Esta disciplina ha posibilitado en los años a que los colaboradores consigan integrarse como equipo y puedan alcanzar los objetivos que se establecen en la organización.

\section{PROPUESTAS DEL CONSEJO ACADÉMICO DE VINCULACIÓN DEL PROGRAMA DE COMUNICACIÓN Y MEDIOS DIGITALES DE LA UNIVERSIDAD POPULAR AUTÓNOMA DEL ESTADO DE PUEBLA}

Durante el mes de marzo del año 2018 fue revisado por el Consejo Académico de Vinculación del Programa de Comunicación y Medios Digitales de la Universidad Popular Autónoma del Estado de Puebla (Upaep) el plan de estudios de la licenciatura con el objetivo de hacer observaciones generales del mismo, así como de cada una de las áreas en las que se pueden desarrollar profesionalmente los egresados (periodismo, investigación de la comunicación, comunicación digital y comunicación corporativa), a continuación se presentaron las recomendaciones hechas por el Consejo como una forma de entender la manera en que es conceptualizada, enseñada y aplicada tanto la comunicación como la comunicación organizacional dentro de la Upaep.

Primeramente, que se propuso hacer un análisis general sobre el plan de estudios de la licenciatura para saber cómo es visto tanto por la institución como por los académicos. Así, una de las primeras preguntas que surge es el porqué del nombre de la licenciatura, lo anterior, haciendo referencia al hecho de que dentro de la carrera se presentan diferentes posturas que apelan al generalismo de la disciplina lo que no supone la consolidación en una sola de ellas tal y como se expresa en el nombre de la licenciatura, derivado de la discusión anterior se llega al cuestionamiento "¿qué es lo que quiere lograr la universidad por medio del programa de comunicación?".

Después, se analizaron cuestiones relativas al contenido del plan de estudios. Un punto importante a resaltar es el hecho de que su propósito fue y es el incluir la teoría y la práctica en cada una de las asignaturas que lo integran. Para que lo anterior se pueda llevar a cabo, es necesario contar con una planta docente que sea capaz de afrontar esta responsabilidad, por lo que se vuelve necesario conocer y mantener actualizado no solo el currículum de los profesores, sino también saber cuántos de ellos son de tiempo completo y además realizan investigación, así como cuántos son maestros de hora clase. Finalmente, se expresa la necesidad de apuntalar la parte de investigación dentro del programa, ya que no se encuentra formalizada, pero se vienen realizando esfuerzos para logarlo como en el caso de las asignaturas de comunicación corporativa en las que se realizan proyectos abarcando temáticas que permiten la intervención en organizaciones de diferente naturaleza.

Por último, se centran en cuestiones más generales que afectan no solo la enseñanza sino también el desarrollo de los estudiantes de la licenciatura, entre ellas se encuentran las siguientes: la falta de líneas terminales que existían en los planes anteriores si existían, así como la ausencia de asignaturas relacionadas a la planeación estratégica que pueden impactar directamente en el desarrollo de las competencias profesionales de los estudiantes de la carrera. Una vez que se 
analizaron los aspectos generales del plan de estudios se trabajó en cada una de las áreas en las que pueden desarrollarse profesionalmente los egresados, en este caso en particular se va a hablar únicamente de los temas que fueron abordados en la mesa de trabajo de comunicación corporativa. Hay que mencionar que la discusión se estructuró alrededor de cuatro grandes preguntas que sirvieron para saber dónde se encuentra la disciplina en este momento y cuáles tendencias que se están siguiendo, qué actividades de vinculación benefician a la licenciatura y a los educandos, para finalmente determinar qué habilidades y conocimientos deben ser desarrolladas en los alumnos.

Así las tendencias en el área de la comunicación organizacional se dirigen hacia el fortalecimiento de las competencias verbales y no verbales, el desarrollo de la investigación, la inclusión de la ética y de la responsabilidad social como los ejes transversales que guíen las acciones de los profesionales que se desarrollen en esta rama de la comunicación; todo lo anterior debe estar englobado en la cultura y en los objetivos de la organización, para que entonces se pueda hablar de una comunicación corporativa y estratégica que lleve al planteamiento y desarrollo de un plan de comunicación a medida de cada organización. Para que los egresados sean capaces de insertarse en esta nueva realidad y puedan resolver las problemáticas que se presentan en el área de comunicación corporativa, es necesario que durante su desarrollo y preparación en la universidad se les ayude a perfeccionar las habilidades que les permitan dar una lectura adecuada del negocio para que puedan hacer mediciones, traducciones, trabajen con los cambios generacionales y de paradigmas, que puedan comprender entornos multiculturales donde la inclusión, la inmediatez, la reputación y el diálogo son indispensables. Finalmente, se vuelve necesario que los egresados cuenten con ciertos conocimientos que sean la base de su desempeño profesional. De acuerdo al Consejo Académico, las áreas más importantes son: redacción, cultura, finanzas, historia, política, economía, estadística, capacidad para construir una realidad y saber cómo va a ser medida por medio del desarrollo de un pensamiento crítico que les permita llegar a un punto de equilibrio entre el valor y el costo de la comunicación en el mercado al relacionarse con otras disciplinas que le permiten mayor crecimiento y desarrollo, tales como la publicidad, los recursos humanos o el desarrollo organizacional.

\section{DISCUSIÓN Y CONCLUSIONES}

La comunicación organizacional en México se ha consolidado en la historia como una disciplina cuyas tendencias han variado, pero siempre sustentadas desde la investigación. En su aplicación, el especialista debe de poner en práctica las competencias verbales y no verbales, la ética, la responsabilidad social, además de tomar en cuenta a la cultura organizacional como la guía que le permita alcanzar los objetivos planteados por la organización; lo anterior, pone en evidencia el hecho de que la CO ha de ser vista de manera integral y no reduccionista.

El estudio de esta disciplina en México se ha enfocado en la heterogeneidad de las organizaciones, que van desde las grandes multinacionales tanto de origen nacional como extranjera hasta las pequeñas y medianas empresas; lo anterior hace indiscutible el hecho de que el estudio de los fenómenos organizacionales en nuestro país ha sido abordado desde muchas perspectivas que han llevado al país a un perfeccionamiento de la disciplina que ha permitido a los investigadores y especialistas crear nuevas ideas, que pueden ser aplicadas a productos, servicios, procesos y formas organizacionales. Para que esto pueda ser llevado a cabo, es necesario que se cuente con un sistema de gestión del conocimiento que pueda ser puesto en práctica con el objetivo de que sus miembros sean capaces de crear, transferir e integrar conocimientos al servicio común, en que se pueda asegurar un diálogo abierto y argumentativo entre los miembros y sus partes.

Así es como este diálogo ha permitido el desarrollo de planteamientos teóricos importantes. Dos casos que vamos a mencionar son los de Rebeil Corella y de Nosnik Ostrowiak. Para el primero, la CO debe ser vista desde una perspectiva de comunicación integral que al interior de las organizaciones se traduce en un proceso de índole social que reside en la producción, el procesamiento y el intercambio de información, que internamente y desde un sistema económico, político, cultural o social se 
da a la tarea de hacer fluir y acelerar mensajes e interacciones entre todos sus grupos de interés, desde tres puntos: público interno y externo, comunicación corporativa y relaciones públicas, y la comunicación mercadológica, que anteriormente se estudiaban por separado. Finalmente, la autora menciona que la Comunicación Organizacional se vuelve una herramienta importante de mejora de las problemáticas en las organizaciones y puntualiza que no es una solución única o exclusiva, ya que permite diseñar estrategias que pueden ser aplicadas a cualquier organización con independencia de su tamaño o naturaleza.

Pero Nosnik Ostrowiak considera que la CO es una noción más profunda que debe cultivarse a partir de la teoría de sistemas, es decir, que se vuelve indispensable ver las organizaciones como un sistema donde cada una de sus partes tiene una función importante que impacta al todo. Además, nos explica que requiere ser abierta y evolucionar para no mantenerse rígida y adaptarse a la realidad y al contexto de las organizaciones, también debe ser multidireccional e instrumental, oportuna, dirigida a los públicos con los que se encuentra relacionada la organización, formal, informal, ascendente, descendente, así como hacer uso de diferentes herramientas para lograrlo. Este autor también menciona que la CO debe partir de la cultura que rige cada sistema, ya que va a ser lo que posibilite la mejora continua en las organizaciones, no con la intención de juzgar, sino de favorecer a quienes integran y se vinculan con la organización.

Finalmente, para integrar el desarrollo que ha tenido la CO en México con el progreso de los planteamientos teóricos, se decidió tomar en cuenta las propuestas del Consejo Académico de Vinculación del Programa de Comunicación y Medios Digitales de la Universidad Popular Autónoma del Estado de Puebla como una manera de comprender de que forma este trabajo académico trasciende en el modo de cómo es conceptualizada, enseñada y aplicada tanto la comunicación como la comunicación organizacional dentro de las universidades en México.

Este análisis se dividió en cuatro ejes que responden a cuestionamientos importantes planteados no solo por la universidad, sino por los académicos y los egresados de la licenciatura en comunicación. La primera pregunta fue "¿Cuáles son las tendencias en ésta área de la comunicación?", y fueron las siguientes: competencias verbales y no verbales, la investigación, el uso y aplicación de la ética, la responsabilidad social, la cultura organizacional, alineación con los objetivos del negocio y la relación con los medios. La segunda pregunta fue “¿Qué habilidades debemos desarrollar en nuestros estudiantes?”; en el consejo se consideraron las siguientes: medir, leer el negocio, traducir, cambio de paradigmas, multiculturalidad, inclusión, inmediatez, reputación integral y el diálogo por encima del control. La tercera pregunta fue "¿Qué conocimientos se debe tener como base de su desempeño profesional?"; y se mencionan los siguientes: redacción, cultura, finanzas, historia, política, ciencias sociales, economía, estadística, pensamiento crítico, construcción de la realidad, el valor de la comunicación en el mercado y el buen generalismo. Por último, se busca responder a la siguiente pregunta "¿Con qué otras unidades, disciplinas o profesiones se relaciona recíprocamente nuestra área de la comunicación?", estas fueron publicidad, recursos humanos y desarrollo organizacional.

Finalmente, podemos decir que el desarrollo que ha tenido la CO como disciplina en México se ve reflejado tanto en los aportes teóricos como en el trabajo que se ha desarrollado en la práctica por los especialistas; lo anterior nos habla no solo de la importancia que tiene esta área de la comunicación, sino de la preocupación que tanto los académicos como las instituciones han mostrado en fortalecerla con el objetivo de que se gane un lugar no solo dentro del ámbito académico, sino al interior de las organizaciones, para que sea vista como una herramienta que les permita cumplir con sus objetivos organizacionales.

\section{REFERENCIAS}

ARELLANO REINOSO, Vanessa Tatiana. Implementación de un plan de inducción y re inducción tendiente a que permita que el personal se identifique con los objetivos organizacionales. 2014. Trabalho de Conclusão de Curso (Bacharelado em Psicologia Industrial) - Facultad de Ciencias Psicologicas, Universidad Central del Ecuador, Quito, 2014. 
DE LA PEZA CASARES, María del Carmen. Los estudios de comunicación: disciplina o indisciplina. Comunicación y Sociedad, Guadalajara, n.20, p.11-32, 2013. Disponível em: https://bit.ly/2M2vYeD. Acesso em: 18 jul. 2019.

GUZMÁN PAZ, Vanessa. Comunicación Organizacional. México, DF: Red Tercer Milenio, 2012.

KARAM, Tanius; CAÑIZÁLEZ, Andrés. Veinte formas de nombrar a los medios masivos. Introducción a enfoques, modelos y teorías de comunicación social. San Cristóbal: Universidad de Los Andes, 2010. (Colección de textos de la comunicación).

NOSNIK OSTROWIAK, Abraham. Teoría de la comunicación productiva: exploraciones más allá de la retroalimentación. Santa Fe: Homo Sapiens Ediciones, 2015.

PEIRÓ SILLA, José Marñia; BRESÓ PEIRÓ, Irene. La comunicación en las organizaciones:

una aproximación desde el modelo de análisis multifacético para la gestión y la intervención organizacional (modelo Amigo). Persona, Lima, n.15, p.41-70, 2012.

PÉREZ CASTREJÓN, Eva María. La ética y la responsabilidad social organizacional. Conceptos fundamentales en la formación y desarrollo profesional de los especialistas de la comunicación organizacional de México y España. Huixquilucan: Universidad Anáhuac México, 2017.

QUERIS ROJAS, Mónica; ALMIRALL CABRERA, Anais; CAPOTE GARCÍA, Lester; ALFONSO ROBAINA, Daniel. Diagnóstico del proceso de comunicación organizacional. Caso de estudio QUIMEFA. Ingeniería Industrial, La Habana, v.33, n.2, p.161-174, 2012.

REBEIL CORELLA, María Antonieta. La comunicación organizacional en las organizaciones privadas y públicas. Organicom, São Paulo, v.3, n.4, p.40-57, 2006.

REBEIL CORELLA, María Antonieta; RUIZSANDOVAL RESÉNDIZ, Celia. El poder de la comunicación en las organizaciones. México, DF: Plaza y Valdés, 2000.

RODRÍGUEZ OLAYA, Reyles Jesús; DE LA ROSA ALBUQUERQUE, Ayuzabet; CONTRERAS MANRIQUE, Julio César. Organización e innovación en bibliotecas: el caso de la BDCV de El Colegio de México. Revista Ciencias Estratégicas, Medelín, v. 21, n.29, p.127-152, 2013.

TEJEDA PALACIOS, Luis. Gestión de la imagen corporativa: creación y transmisión de la identidad de la empresa. Bogotá: Editorial Norma, 1987.

Artículo recibido el 08.02.2019 y aprobado el 18.07.2019. 